\title{
Quasi-static PEEC planar solver using a weighted combination of 2D and 3D analytical Green's functions and a predictive meshing generator
}

\author{
Saiyd Ahyoune ${ }^{\mathrm{a}, *}$, Javier Sieiro $^{\mathrm{a}}$, Tomás Carrasco ${ }^{\mathrm{a}}, \mathrm{Neus}_{\text {Vidal }}^{\mathrm{a}}$, José M. López-Villegas ${ }^{\mathrm{a}}$, Elisenda Roca ${ }^{\mathrm{b}}$, Francisco \\ V. Fernández ${ }^{\mathrm{b}}$ \\ ${ }^{a}$ Grup de Radiofreqüència-Universitat de Barcelona, Barcelona, Spain \\ ${ }^{b}$ Instituto de Microelectrónica de Sevilla, IMSE-CNM, CSIC and Universidad de Sevilla, Seville, Spain
}

\begin{abstract}
In this work, a quasi-static implementation of the partial element equivalent circuit (PEEC) method for the analysis of planar radiofrequency $(\mathrm{RF})$ and microwave $(\mathrm{uW})$ components is proposed. The procedure is divided in three parts. First, an alternative PEEC formulation based on energy concepts is described. Second, a smart mesh generator is developed in order to provide an accurate solution at minimum computational costs, taking into account both geometry and device physics as metrics for the correct sizing of mesh elements. And third, a weighted combination of the 2D and 3D quasistatic Green's functions (GF) is proposed for extending the valid frequency range of the quasi-static approximation. It is shown that the $3 \mathrm{D}-\mathrm{GF}$ is very accurate at low frequency, whereas the $2 \mathrm{D}-\mathrm{GF}$ is more suitable at higher frequencies. Numerical examples are compared to experimental data for different passive components and technologies in a wide frequency range.
\end{abstract}

Keywords: PEEC, quasi-static approximation, mesh generator, Green's function.

\section{Introduction}

Quasi-static solvers are popular for the modelling of RF passives when the effect of retarded potentials can be ignored. As a rule of thumb, the quasi-static approximation holds if the ratio of the maximum typical distance between two points of the geometry, $\ell$, over the wavelength of the simulated frequency, $\lambda$, is small enough, e.g. $\ell / \lambda<0.05$. Despite this limitation, many different devices can be studied using this formulation. For instance, the behavior of typical spiral inductors can be computed beyond their selfresonant frequency $(\mathrm{SRF})$ [1. Worst cases are related to transmission line-based components, e.g. in the design of a $\lambda / 4$ transformer [2].

In the development of quasi-static solvers, the PEEC method 3 has shown great ability for dealing with the electromagnetic analysis of devices with Manhattan-like layouts [1, 4]. The main feature underlying this outstanding ability is the easy computation of large aspect ratio elements. With the introduction of non-orthogonal elements [5], the method can be applied to other complex layout components, but with the penalty of an increase in computational time. Other interesting PEEC features for the modelling of passive devices are [6]: (i) its model

\footnotetext{
This work was supported by the Spanish Ministry of Science and Innovation under projects TEC2013-40430-R, TEC2017-83524$\mathrm{R}$, and TEC2016-75151-C3-3-R.

* Corresponding author.

Email address: sahyoune@el.ub.edu, ahyoune@gmail.com (Saiyd Ahyoune)
}

order reduction capability, meaning that it can be formulated as a stationary (DC), Lorentz/magneto/electro quasi-static or full-wave spectrum with no extra effort; (ii) its application to multilayered substrates, including their top and bottom boundary conditions, which can be easily implemented using analytical Green's functions; and (iii) its seamless integration into circuit simulators.

Despite these advantages, the practical implementation of quasi-static PEEC solvers for planar RF and $\mathrm{uW}$ circuits has two main challenges. The first deals with the generation of the mesh. To speed up the computation, the mesh must be restricted to a small number of elements. However, current crowding and the skin effect are important phenomena that demand a refined mesh to achieve reasonable solution accuracy [1. The second is related to the maximum simulation frequency. Based on the $\ell / \lambda<0.05$ criterion, only a few components can be analyzed up to its SRF.

In this work, both challenges are addressed and practical solutions are developed. For the mesh generation problem, two meshing techniques are introduced. The first technique deals with the concept of the skin effect, i.e. the current density distribution along the thickness of a metal strip. By means of a semi-analytical formulation, the effect of skin depth on the sheet resistance value is captured with reasonable accuracy. The second technique takes into account, at first order, the influence of the current crowding distribution along the width of a metal strip; thus an $a b$ initio adaptive mesh is generated. To extend the simulation frequency, without compromising accuracy in the low 
frequency regime, a weighted linear combination of the quasi-static $3 \mathrm{D}-\mathrm{GF}$ of the substrate, $G_{3 D}$, with its $2 \mathrm{D}-\mathrm{GF}$ counterpart, $G_{2 D}$, is proposed [7]. The quasi-static $G_{2 D}$ is actually the solution of the transverse electromagnetic (TEM) propagation mode in transmission line problems; thus, it can provide good results in the $\mathrm{GHz}$ range [8, 9]. However, it can neither accurately handle the finite length geometry of the layout, nor high-order propagation modes. On the contrary, the quasi-stationary $G_{3 D}$ is associated with a point charge or an infinitesimal horizontal/vertical current element [10]. In this case, there is no restriction on the geometry, but the description at higher frequency is less accurate due to direct electrical/magnetic couplings in the direction of propagation. With the combination of both GFs, the main self-resonances of passives, including transmission line-based components, are computed accurately, whereas the low frequency behavior is determined correctly.

The paper is organized as follows. In Section 2, an alternative formulation of the PEEC method for linear and time-invariant (LTI) laminates is proposed by applying Hamilton's principle to a multiconductor system. Section 3 deals with the development of the two meshing techniques mentioned above for the implementation of a mesh generator. In Section 4 the limitations of both quasi-static $G_{3 D}$ and $G_{2 D}$ are studied. The criteria for selecting the weights of their linear combination are also explained. In Section 5, the developed PEEC solver is applied to the computation of the electromagnetic behavior of a set of different devices: a finger capacitor, an inductor and a microwave filter. Finally, the conclusions of the work are presented in Section 6.

\section{PEEC formulation}

Planar components can be understood as multiconductor systems formed by a combination of metal strips and patches. Under the excitation of this system by an external source, current and charge density distributions will arise and produce an electromagnetic response. To numerically compute these distributions using the PEEC method, conductor domains are partitioned in small cells, as shown in Fig. 1. Inside each cell, charge and current can be considered uniformly distributed. One mesh partition is set for currents, with a total number of $M$ cells, and another one for charges, formed by $N$ cells. The approximated solution is obtained by solving the unknown $M$-currents and $N$-charges.

To find the required $M+N$ equations, two main steps are conducted in the PEEC formulation [3]. In its classical approach, the first step comprises the application of Galerkin's method to the mixed potential integral equation. Then, a Kirchhoff's voltage law (KVL) equation is obtained for each current cell. Alternatively, the same equation can be obtained using the Lagrangian energy description of a multiconductor system, $\mathcal{L}$. The motivation for this treatment is the increasing interest in Lagrangian
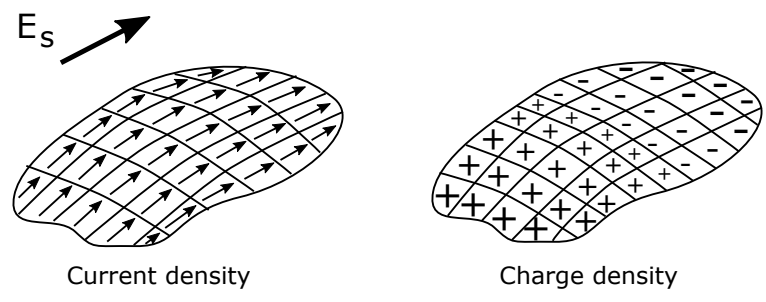

Fig. 1: Numerical approximation.

methods applied to device and circuit modeling [11, 12. To the best of the authors' knowledge, the first time that this interpretation was applied to the PEEC method can be found in [10]. For completeness regarding implementation of the solver, this alternative formulation is briefly explained in Section 2.1.

In the second step, the continuity equation is applied to each charge cell; thus, a Kirchhoff's current law (KCL) equation appears. Actually, this equation sets the relationship between charge and current meshes. To facilitate the partial element stamping inside the system equations matrix, the solution adopted in the classical PEEC formulation is to force both meshes to be each other's dual.

It should be noted that retarded potentials are neglected in the following procedure due to the quasi-static restriction. Therefore, neither radiation effects, nor highorder propagation modes, can be considered in the simulation of a device. In addition, the substrate materials are LTI.

\subsection{Kirchhoff's voltage law}

The Lagrangian operator, $\mathcal{L}$, is defined as the difference between the electrical energy and magnetic co-energy. The electrical energy of the charge mesh, $W_{e}$, is given by

$$
W_{e}=\frac{1}{2} \bar{q}^{T}[P] \bar{q},
$$

where $\bar{q}$ is a column vector comprising the charges $q_{i}$ at each cell, $\bar{q}^{T}$ as its transpose, and $[P]$ the partial scalar potential matrix. The coefficients of $[P]$ are defined by

$$
P_{i j}=\frac{1}{v_{i}} \frac{1}{v_{j}} \int_{v_{i}} d^{3} \bar{r} \int_{v_{j}^{\prime}} \overline{\bar{G}}\left(\bar{r}, \bar{r}^{\prime}\right) d^{3} \bar{r}^{\prime},
$$

where $\overline{\bar{G}}\left(\bar{r}, \bar{r}^{\prime}\right)$ is the scalar potential Green's function related to the layered substrate, and $v_{i}$ and $v_{j}$ are the volumes of charge cells $i$ and $j$, respectively.

The magnetic co-energy, $W_{m}$, is defined as

$$
W_{m}=\frac{1}{2} \bar{I}^{T}[L] \bar{I}
$$

where $\bar{I}$ is a column vector comprising the currents $I_{i}$ at each cell, $\bar{I}^{T}$ as its transpose, and $[L]$ the partial inductance matrix. The coefficients of $[L]$ are given by

$$
L_{i j}=\frac{1}{A_{i} A_{j}} \int_{v_{i}} \int_{v_{j}^{\prime}} \hat{u}_{i} \cdot \hat{u_{j}} \overline{\bar{K}}\left(\bar{r}, \bar{r}^{\prime}\right) d v d v^{\prime},
$$




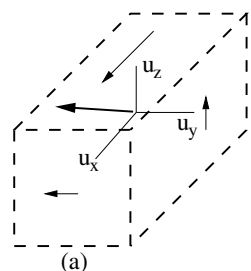

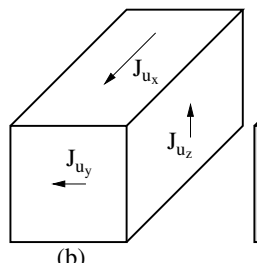

(b)

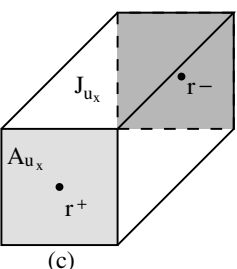

Fig. 2: Geometric definition of a current cell: (a) current density $\bar{J}$ inside a volume; (b) projection of the components of $\bar{J}$; (c) current cell in the $\hat{u_{x}}$ direction. Current cells in the $\hat{u_{y}}$ and $\hat{u_{z}}$ are defined in the same way as (c).

where $v_{i}$ and $v_{j}$ are now the volumes of the current cells; $\hat{u}_{i}$ and $\hat{u}_{j}$ are the current directions; the cross-section of each current cell is represented by $A_{i}$ and $A_{j}$; and $\overline{\bar{K}}\left(\bar{r}, \bar{r}^{\prime}\right)$ is the vector potential Green's function of the layered media. As shown in Fig. 2, the three components of the current density vector $\bar{J}$ are split into three orthogonal cells.

Using Eq. (1) and Eq. (3), the Lagrangian of the multiconductor system, $\mathcal{L}=W_{m}-W_{e}$, is written as follows

$$
\mathcal{L}=\frac{1}{2} \bar{I}^{T}[L] \bar{I}-\frac{1}{2} \bar{q}^{T}[P] \bar{q}=\frac{1}{2} \overline{\dot{q}}^{T}[L] \overline{\dot{q}}-\bar{q}^{T}[P] \bar{q}
$$

where $\dot{q}=d q / d t=I$. Now, Kirchhoff's voltage law is obtained using the non-conservative equation of Hamilton's principle [12, i.e.,

$$
\frac{d}{d t}\left(\partial_{\dot{q}_{i}} \mathcal{L}\right)-\partial_{q_{i}} \mathcal{L}+\partial_{\dot{q}_{i}} R=V_{k}^{s},
$$

where $V_{k}^{s}$ is the applied external voltage source, and $R$ is the Rayleigh dissipation factor that, for circuit analysis, is the loss due the resistive elements $R_{i}$

$$
R=\frac{1}{2} \sum_{i}^{N} R_{i}\left(\dot{q}_{i}\right)^{2}
$$

After some algebraic manipulation of Eq. (6) and using the Fourier transform $d / d t=j \omega$, the resulting KVL equation at each current cell $k$ is given by

$$
V_{k}^{s}=R_{k} I_{k}+j \omega \sum_{l}^{M} L_{k l} I_{l}+\phi\left(r_{k}^{+}\right)-\phi\left(r_{k}^{-}\right) .
$$

This equation asserts that the application of an external voltage source to a current cell equals the voltage decay due to losses, plus the decay due to the magnetic interaction, plus the scalar voltage difference between the input/output current cell cross-sections.

\subsection{Kirchhoff's current law}

The remaining $N$ equations are set with the help of the continuity equation for charge conservation, i.e.

$$
\bar{\nabla} \bar{J}(\bar{r})+j \omega \rho(\bar{r})=0
$$

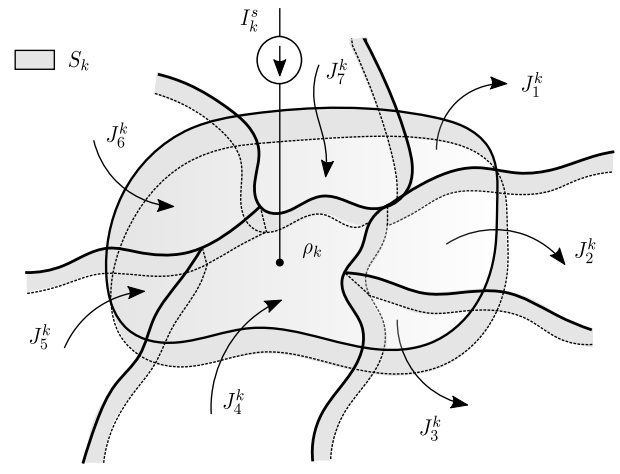

Fig. 3: Charge and current densities through cell $k$ boundary surface $S_{k}$

where $\bar{J}(\bar{r})$ and $\rho(\bar{r})$ are the current and charge density distributions, respectively. Fig. 3 depicts the relationship between a charge cell $k$ and its neighboring current cells. According to Eq. (9) and Fig. 3, the displacement current, $j \omega \rho_{k}$, must equal the total sum of input and output of nearby conduction currents, $I_{m}^{I N_{k}}$ and $I_{m}^{O U T_{k}}$, plus any external current source, $I_{k}^{s}$, i.e.

$$
j \omega \sum_{l=1}^{N} C_{k l} \phi\left(r_{l}\right)=I_{k}^{s}+\sum_{m=1}^{N_{I N_{k}}} I_{m}^{I N_{k}}-\sum_{m=1}^{N_{O U T_{k}}} I_{m}^{O U T_{k}}
$$

This expression uses the relationship $q_{k}=\sum_{l=1}^{N} C_{k l} \phi\left(r_{l}\right)$, where $C_{k l} \equiv P_{k l}^{-1}$ is the partial capacitance matrix. At this point, a comment must be made about the definition of the scalar potential $\phi\left(r_{l}\right)$. In Eq. (8), it is related to the input/output cross-sections of the current cell; but this definition is not the same in Eq. 10, where it is considered as the mean value inside the charge cell volume. In most PEEC solvers, these two concepts are merged by making the current and the charge meshes act as each other's dual. Nonetheless, other meshing configurations compatible with Eq. (10) are also possible [13, such that $1 \mathrm{D}$ and $2 \mathrm{D}$ current density elements can be assembled by means of Eq. (8), allowing the use of unstructured meshes.

Finally, the complete system of harmonic equations, Eq. (8) and Eq. (10), can be written in matrix form as

$$
\left[\begin{array}{l}
\bar{V}^{s} \\
\bar{I}^{s}
\end{array}\right]=\left[\begin{array}{cc}
\overline{\bar{R}}+j \omega \overline{\bar{L}} & \overline{\bar{D}}^{T} \\
\overline{\bar{D}} & j \omega \overline{\bar{C}}
\end{array}\right]\left[\begin{array}{l}
\bar{I} \\
\bar{\phi}
\end{array}\right]
$$

where $\bar{I}$ and $\bar{\phi}$ are the unknown currents and scalar potentials, $\overline{\bar{R}}$ is the resistance diagonal matrix, $\overline{\bar{L}}$ is the partial inductance matrix, $\overline{\bar{C}}$ is the partial capacitance matrix, $\overline{\bar{D}}^{T}$ is the transpose of $\overline{\bar{D}}$ that describes the connection of branches to their respective nodes, and $\bar{V}^{s}$ and $\bar{I}^{s}$ are the external voltage and current sources.

\section{Mesh generator}

In most planar circuits, the current density distribution, $\bar{J}(\bar{r})$, is dominated by the skin effect. Without any 


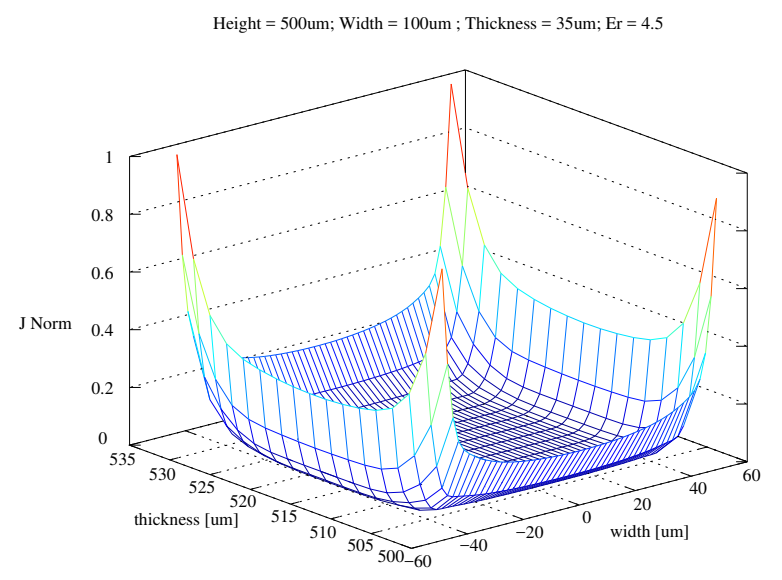

Fig. 4: Simulated cross-section $\bar{J}(\bar{r})$ distribution for a microstrip line placed on a $0.5 \mathrm{~mm}$ FR4 substrate at $1 \mathrm{GHz}$.

other knowledge about $\bar{J}(\bar{r})$, current cells must be sized according to the skin depth value, $\delta$, for an accurate numerical description of this phenomenon. For instance, the value of $\delta$ for copper at $1 \mathrm{GHz}$ is about $2 \mu \mathrm{m}$, which translates into a dense mesh. The penalty for this accuracy is a slow run time. Fig. 4 shows the simulated $\bar{J}(\bar{r})$ of a microstrip line in its cross-section. On the basis of this solved profile, it is clear that current cells could be sized according to the gradients of $\bar{J}(\bar{r})$. Thus, it is only necessary to have a refined mesh at the edges of the microstrip. In differential solvers, this feature is accomplished with the implementation of an ad hoc adaptive mesh technique. This is an iterative solution procedure where the mesh is refined at each step using the solution of the actual iteration as the control sizing function. Although accuracy is highly improved, computation speed can be worsened due to the iterative nature of the technique. On the contrary, the point of view adopted in this work is that the mesh generator must have an $a b$ initio predictive method to figure out the correct size of a cell before any complete solution of the simulation problem is available. Therefore, iterations are avoided. In this sense, the mesh generator must be physics- and layout-aware.

\subsection{Current mesh generator}

In the most general case of a planar device, such the one shown in Fig. 5 , its geometry can be broken into metal strips. Therefore, the current density distribution for each strip has two components:

(i) The eddy current distribution of the own-magnetic field due to the impressed current in the strip, which shapes the skin effect behavior.

(ii) The eddy current distribution of the external-magnetic field due to the magnetic coupling with the remaining strips, which shapes the crowding behavior.

Given the above, the proposed current mesh generator employs two techniques. The first one uses a semianalytical

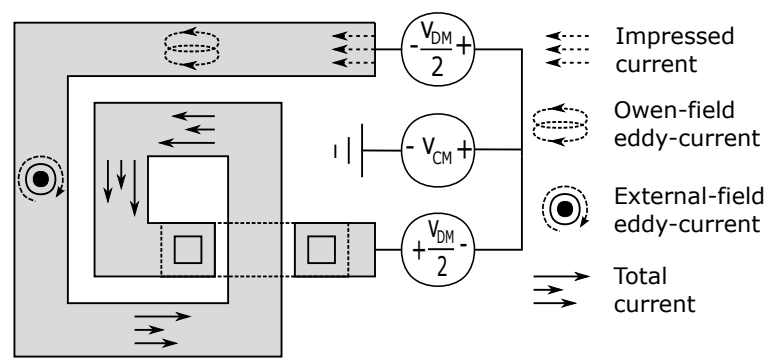

Fig. 5: Physical interpretation of current distribution in inductors.

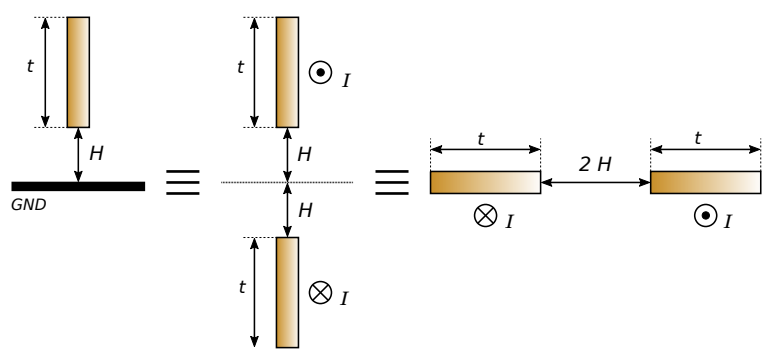

Fig. 6: Equivalent problem for the computation of $\bar{J}$ in microstrip configuration.

formula that takes into account the impact of $\delta$ on the sheet resistance value of a flat conductor. Using a simple PEEC model of a metal strip, the second technique predicts the influence of the current crowding distribution along the width of the strip. These procedures are explained below.

\subsubsection{Skin effect model}

Commonly, the skin effect in metal strips, defined as the current distribution across the thickness, is mostly modeled as a surface impedance, $Z_{S}$. The major impact of the skin effect is the change in the actual sheet resistance of the strip, $R_{S}$, defined as

$$
R_{S}=R_{S_{D C}} \cdot \frac{t}{\delta\left(1-e^{-t / \delta}\right)}
$$

where $R_{S_{D C}}$ is the DC sheet resistance, $t$ is the thickness of the conductor, and $\delta$ is the skin depth at the frequency of interest. Intrinsically, the use of $Z_{S}$ is linked to a symmetric distribution of $\bar{J}(\bar{r})$ along the thickness of the strip. However, when in close proximity to a ground plane [14, the symmetry of the distribution pattern is broken and Eq. 12 is no longer valid. To solve this modeling problem, different techniques have been developed that can be classified as exponential, general surface impedance, general boundary impedance, and volume filament models. A nice review of these techniques can be found in [15. Exponential models are faster but more limited, such the one described by Eq. 12, whereas volume filament methods are the most accurate but with a slower run time, e.g. [16].

The proposed solution for our PEEC solver is an exponential model that modifies Eq. (12) with the introduction 


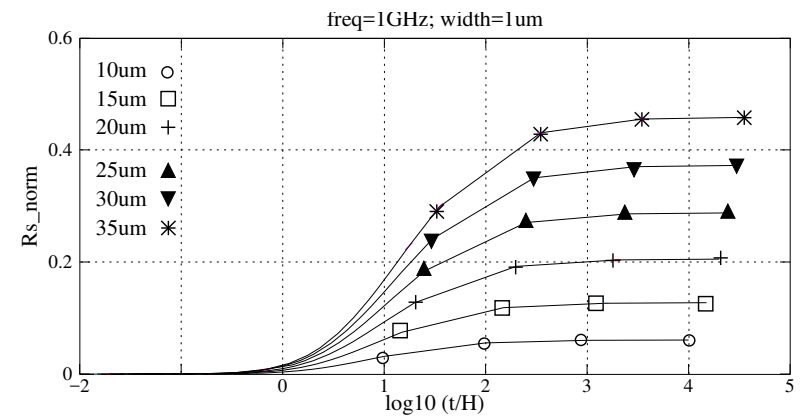

Fig. 7: Normalized loss of the strip vs $(t / H)$.

of an additional factor. This factor needs to be calculated only once for each technology under consideration, where the thickness of the strip, $t$, and the distance to the ground plane, $H$, are fixed. The procedure is based on the solution of the equivalent problem shown in Fig. 6. The ground plane can be substituted by the image of the strip; thus, the actual problem is the computation of the losses related to two-coupled metal strips, separated by a distance $2 H$, driven differentially, and having the same reversed widththickness dimensions of the original problem. In this problem, the width of the strip, $w$, is fixed to a small value, e.g. $w=1 \mu \mathrm{m}$ for a maximum simulation frequency of 10 $\mathrm{GHz}$ using copper as the conductor material. Fig. 7 shows the results of the normalized loss

$$
R_{S_{\text {norm }}}=\frac{R_{S}(t / H)}{R_{S}(t / H \rightarrow 0)}-1
$$

for different values of thickness. Notice that the independent variable in this plot is $t / H$. An interesting point is that all traces have the same functional form, which is given by

$$
f(t / H)=\frac{A(t / H)^{2}}{1+B(t / H)^{2}} .
$$

The values of $A$ and $B$ are dependent on frequency. Therefore, the procedure must be repeated according to the intended frequency range. Thereafter, the values of $A$ and $B$ can be stored inside the PEEC solver. Another interesting fact about Fig. 7 is that Eq. (12) holds for $H>2 \cdot t$. Otherwise, the sheet resistance is modified as

$$
R_{S}=R_{S_{D C}} \cdot \frac{t}{\delta\left(1-e^{t / \delta}\right)} \cdot\left(1+\frac{A(t / H)^{2}}{1+B(t / H)^{2}}\right)
$$

\subsubsection{Current crowding effect}

Current crowding is related to the redistribution of the current density in a given layout location due to the magnetic interaction with the remaining parts of the layout. In [17], an ab initio current crowding prediction algorithm was proposed. Recently, it was improved in 10 . by including the effect of the autoinductance of the metal strip. For the completeness of the proposed PEEC solver, this algorithm consists of the realization of a simple PEEC model

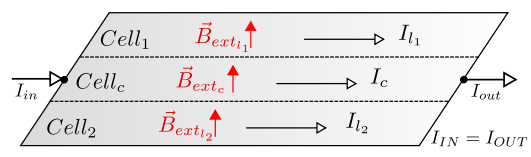

(a)

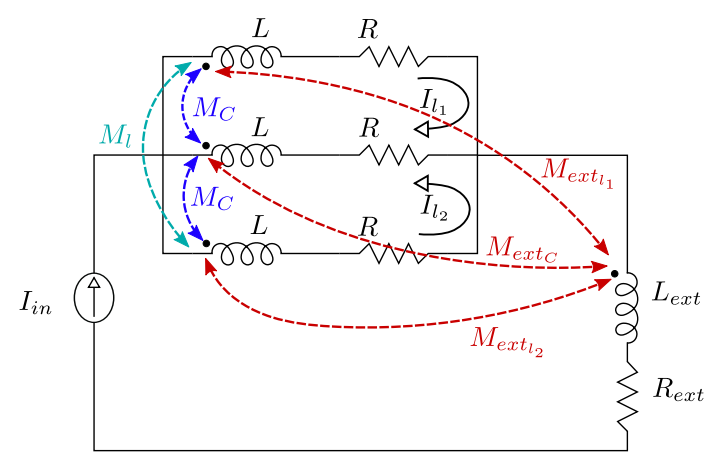

(b)

Fig. 8: (a) Simple mesh of a metal strip, (b) equivalent circuit model.

Table 1: Number of cell divisions along the width.

\begin{tabular}{l|c}
\hline$P_{\text {ACloss }} / P_{\text {DCloss }}=x$ & $\sharp$ cells \\
\hline$x<1.5$ & 3 \\
$1.5<x<2.5$ & 5 \\
$2.5<x<4$ & 7 \\
$4<x$ & 9 \\
\hline
\end{tabular}

of the strip that must be meshed across its width. As shown in Fig. $8(\mathrm{a})$, the strip is divided into three cells $l_{1}$, $l_{2}$ and $c$, carrying their respectives currents $I_{l_{1}}, I_{l_{2}}$ and $I_{c}$. The partial element circuit model is sketched in Fig. 8(b). Each cell has its own impedance $j \omega L+R$; cells are magnetically coupled to each other through $M_{c}$ and $M_{l}$; and the magnetic influence of the remaining strips of the layout is represented by $M_{\text {ext }_{l_{1}}}, M_{\text {ext }_{l_{2}}}$ and $M_{\text {ext }}$. Parameters $L_{\text {ext }}$ and $R_{\text {ext }}$ are related to the self impedance of the remaining parts of the device.

The strategy for sizing the current cells adequately is based on the computation of the AC/DC power loss ratio of the strip, $x$, which can be obtained by solving $I_{l_{1}}$ and $I_{l_{2}}$ in Fig. 8(b). Based on previous experience, the number of cells across the width is defined in Table 1 according to $x$. These cells are non-uniformly distributed using a $\delta$ criterion.

\subsection{Charge mesh generator}

In [18, it was shown that the stability of a PEEC solver is relatively sensitive to the charge mesh distribution because it involves a matrix inversion in Eq. (11), i.e., $[C]=\left[P^{-1}\right]$. For this reason, the layout should be meshed according to the expected charge distribution. This requirement can be met using two techniques: (i) a projection meshing, explained in [18; and (ii) forcing a higher mesh density at the conductor boundaries, where charges are accumulated. Nonetheless, for the latter method, the 
mesh density at the edges can be relaxed when the computation of partial coefficients is performed with analytical expressions. To understand this statement, the simulated charge density of a squared patch using a uniform mesh is represented in Fig. 9(a), whereas a non-uniform edge mesh is shown in Fig. 9(b). Clearly, the obtained solutions are completely different. Despite this difference, the obtained capacitance value is similar, i.e., $4.01 \mathrm{fF}$ for the uniform mesh and $4.06 \mathrm{fF}$ for the non-uniform mesh. The reason for this similarity is that the partial elements are actually a measure of the energy associated with a pair of cells, as shown in Section 2, Keeping this result in mind, the size of charge cells is set according to the sizes of their neighboring current cells.

\section{Frequency range extension of quasi-static solvers}

\subsection{Analytical 3D quasi-static Green's functions}

As shown in Eq. (2) and Eq. (4), the computation of $P_{i j}$ and $L_{i j}$ involves a double volume integral, and the knowledge of $\overline{\bar{G}}\left(\bar{r}, \bar{r}^{\prime}\right)$ and $\overline{\bar{K}}\left(\bar{r}, \bar{r}^{\prime}\right)$. In this section, the study is restricted to isotropic materials; thus, $\overline{\bar{G}}\left(\bar{r}, \bar{r}^{\prime}\right)=$ $G\left(\bar{r}, \bar{r}^{\prime}\right)$ and $\overline{\bar{K}}\left(\bar{r}, \bar{r}^{\prime}\right)=K\left(\bar{r}, \bar{r}^{\prime}\right)$. Compared to numerical methods, the computation of these GFs and the partial coefficients using analytical expressions is more accurate and naturally enforces the passivity of the system matrix in Eq. 11). In addition, the computation time of GFs in numerical approaches can be quite large when the substrate has many layers. However, only a few substrates can be efficiently described using analytical expressions.

Despite the above-mentioned limitation, the framework for this proposed quasi-static PEEC solver is an analytical methodology based on Hankel's transform [10 and a Coulomb gauge. Most available hybrid technologies can be interpreted as a homogeneous LTI dielectric multilayered substrate, as shown in Fig. 10 for an LTCC stack, which is amenable to this methodology. For instance, the next $G_{3 D}$ expression corresponds to a layered substrate with a total dielectric thickness $H$, and an underneath ground plane, i.e.

$$
\begin{aligned}
& G(\rho, z)=\frac{1}{4 \pi \epsilon_{M}}\{ \frac{1}{\sqrt{\rho^{2}+(H-z)^{2}}}-\frac{1}{\sqrt{\rho^{2}+(H+z)^{2}}}+ \\
&+\sum_{l=1}^{\infty}(-1)^{l} k^{l}\left[\frac{1}{\sqrt{\rho^{2}+(H-z+2 H \cdot l)^{2}}}-\right. \\
&\left.\left.\quad-\frac{1}{\sqrt{\rho^{2}+(H+z+2 H \cdot l)^{2}}}\right]\right\}, \quad(16)
\end{aligned}
$$

where $\rho$ is the radial cylindrical coordinate, $\epsilon_{M}=\frac{\epsilon_{L T C C}-\epsilon_{a i r}}{2}$, and $k=\frac{\epsilon_{L T C C}-\epsilon_{a i r}}{\epsilon_{L T C C}+\epsilon_{a i r}}$. In this case, the point charge source and the observation point are located at the interface between the dielectric and the open boundary. Eq. (16) has been arranged such that the following properties are illustrated, a fact that is quite complicated to visualize using numerical techniques:

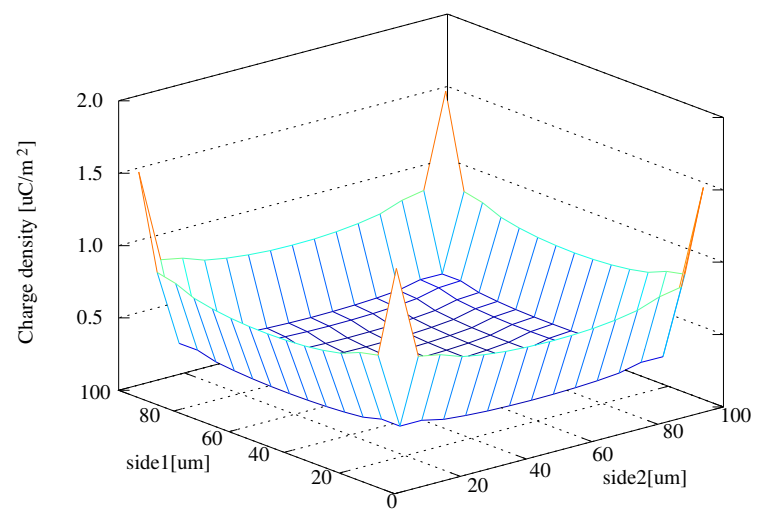

(a)

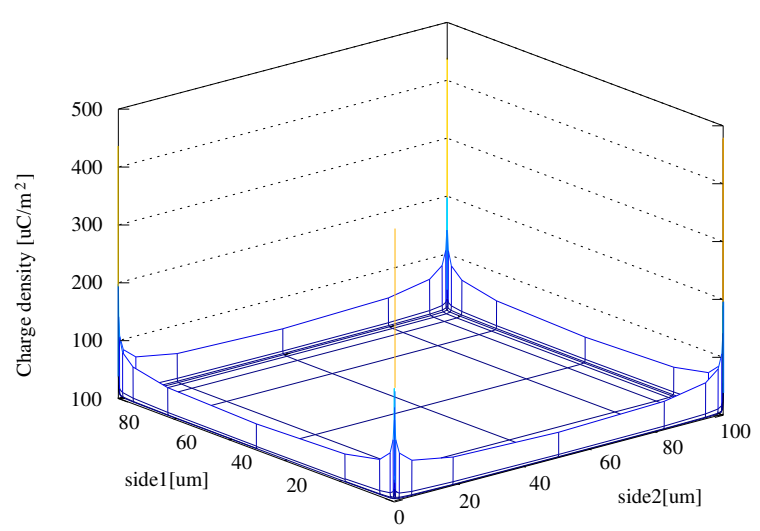

(b)

Fig. 9: Simulation of the charge mesh density of an squared $100 \times$ $100 \mathrm{\mu m}^{2}$ patch: (a) uniform mesh; (b) non uniform mesh.

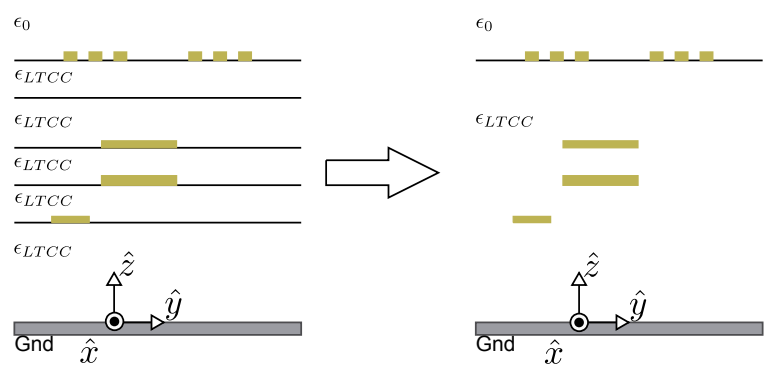

Fig. 10: Collapse of a multilayered LTCC substrate in two dielectric layers. Inside the LTCC dielectric, conductors can be placed anywhere.

- Both terms out of the summation are the solution of the half space with a permittivity equal to $\epsilon_{M}$.

- Each term $l$ of the summation is formed by a charge of value $(-k)^{l}$ located at $2 \cdot H \cdot l$ above the LTCC-air interface and its image through the GND plane, as shown in Fig. 11.

- Charges are grouped in pairs, the charge and its image, thus it is possible to see them as dipoles of value $p=2 H(l+1) \cdot(-k)^{l}$. This interpretation can be very helpful for the development of asymptotic expressions. 


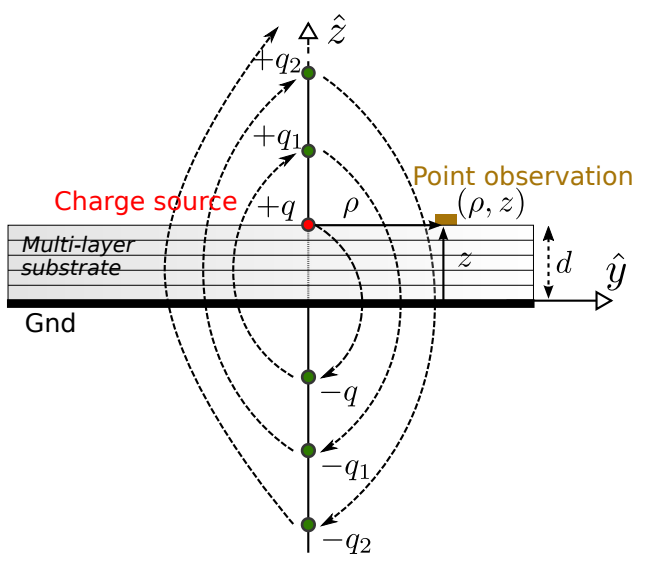

Fig. 11: Image charge situation in LTCC substrate with GND.

- The double volume integral of any term in Eq. 16 has an analytical form defined by the expressions developed by Hoer and Love [19].

The main advantage of this procedure is that partial elements with a large aspect ratio can be evaluated accurately. However, the disadvantage is the limited simulation frequency range due to the quasi-static approximation, i.e., $\ell / \lambda<0.05$.

\subsection{D vs. 3D quasi-static Green's functions}

To explore a plausible extension of the frequency range, note that the quasi-TEM solution of a transmission line (TL) can be found using the $2 \mathrm{D}$ quasi-static equations of the vector potential $\bar{A}$ and the scalar potential $\phi$. The solution of these equations is based on a $2 \mathrm{D}-\mathrm{GF}, G_{2 D}$. In this kind of TL model, the limit of the maximum simulation frequency is the minimum cut-off frequency of the surface-wave, $f_{s}$, and the first high-order wave-guide, $f_{c}$, propagation modes [20], i.e.

$$
f_{s}=\frac{c_{0} \cdot \tan ^{-1}\left(\epsilon_{r}\right)}{\pi H \sqrt{2} \sqrt{\epsilon_{r}-1}} ; \quad f_{c}=\frac{c_{0}}{(2 H+0.8 t) \sqrt{\epsilon_{r}}},
$$

where $c_{0}$ is the speed of light in vacuum, and $\epsilon_{r}$ is the relative permittivity of the dielectric. For instance, the maximum simulation frequency of a microstrip line having $\ell=3 \mathrm{~mm}, w=0.5 \mathrm{~mm}, t=10 \mu \mathrm{m}$ placed on a substrate with $H=0.5 \mathrm{~mm}$ and $\epsilon_{r}=9.28$ is $68.7 \mathrm{GHz}$ using a $G_{2 D}$ function, whereas it is $1.7 \mathrm{GHz}$ for a $G_{3 D}$ function.

To further explore the limitations of $G_{2 D}$ and $G_{3 D}$, the behavior of the previous microstrip line, whose characteristic impedance is $50 \Omega$, was studied using different electromagnetic solvers. The simulation frequency was set to $100 \mathrm{MHz}$; thus, $\ell / \lambda \approx 0.0026$. The employed solvers are listed in Table 2. Except for $G_{3 D}$, where the ports are of the internal type, the remaining methods make use of calibrated ports.

Two lumped and two distributed parameters were chosen to evaluate the performance of the transmission line. The lumped parameters are the equivalent inductance,

$$
L=\operatorname{imag}\left(Z_{\text {diff }}\right) / \omega
$$

Table 2: Simulated transmission line characteristics of a microstrip line $(\ell=3 \mathrm{~mm}, \mathrm{w}=0.5 \mathrm{~mm})$.

\begin{tabular}{c|c|c|c|c}
\hline Method & $\epsilon_{\text {eff }}$ & $Z_{0}$ & $\mathrm{~L}(\mathrm{nH})$ & $\mathrm{C}(\mathrm{fF})$ \\
\hline$G_{2 D}[21]$ & 6.36 & 50.1 & 1.27 & 501 \\
\hline$G_{3 D}[10]$ & 6.38 & 46.7 & 1.18 & 541 \\
\hline \hline MoM-RF [21] & 6.21 & 50.2 & 1.25 & 497 \\
\hline MoM-uW [21] & 6.10 & 49.8 & 1.23 & 496 \\
\hline Sonnet [22] & 5.85 & 49.2 & 1.19 & 492 \\
\hline CST [23] & 6.06 & 48.8 & 1.20 & 505 \\
\hline
\end{tabular}

and the equivalent capacitance,

$$
C=\operatorname{imag}\left(Y_{\text {open }}\right) / \omega,
$$

where $Z_{\text {diff }}$ is the microstrip differential impedance of the line, and $Y_{\text {open }}$ its open-circuit admittance. The distributed parameters are the characteristic impedance, $Z_{0}$, and the effective permittivity, $\epsilon_{\text {eff }}$. These two parameters are related to $L$ and $C$ using the per-unit-length (p.u.l.) inductance, $L / \ell$, and the p.u.l. capacitance, $C / \ell$, as follows

$$
\begin{gathered}
Z_{0}=\sqrt{L / C} \\
\epsilon_{\mathrm{eff}}=L C c_{0}^{2} / \ell^{2}
\end{gathered}
$$

In Eq. 20 and Eq. 21, the lossless TL approach is considered.

Table 2 shows the obtained performance parameters. Starting with $\epsilon_{\text {eff }}$, the two analytical methods, $G_{2 D}$ and $G_{3 D}$, show a similar value. MoM-RF also shows a similar value when compared to analytical solutions; however, small discrepancies are obtained with the remaining solvers, which can be attributed to the finite thickness of the metal strip, the way of calculating the calibrated ports, or the presence of artificial boundary conditions for truncating the model in the case of CST. For $Z_{0}$, the obtained values are close to $50 \Omega$, within a $2 \%$ error bound; but, for $G_{3 D}$, it is $46.7 \Omega$. The reason for this odd behavior is that calibrated port methods eliminate the finite length dependence on $Z_{0}$. By introducing calibrated ports in the $G_{3 D}$ solver, the obtained $Z_{0}$ is $50.3 \Omega$ (not shown in Table 2). However, notice that the introduction of calibrated ports in $G_{3 D}$ actually transforms this method into a $G_{2 D}$ method.

Inspection of the lumped parameters reveals two different behaviors. All commercial tools show a $C$ value closer to $G_{2 D}$ than to $G_{3 D}$. When using a calibrated port in $G_{3 D}$, the obtained $C$ value is $493 \mathrm{fF}$ (not shown in Table 2), which is close to $G_{2 D}$. On the contrary, the obtained values of $L$ vary widely between $G_{2 D}$ and $G_{3 D}$ solutions. Although it might seem that these differences in $L$ are small, they are of utmost importance when dealing with actual devices. For instance, Table 3 presents the main parameters of a $2.0 \mathrm{nH}$ inductor fabricated in a $0.18 \mu \mathrm{m}$ 
Table 3: Electrical parameters of a RFIC inductor in a $0.18 \mu \mathrm{m}$ CMOS technology.

\begin{tabular}{c|c|c|c|c}
\hline Method & $L(\mathrm{nH})$ & $Q_{\max }$ & $S R F(\mathrm{GHz})$ & $R_{D C}(\Omega)$ \\
\hline 3D-GF & 2.09 & 21.3 & 16.80 & 1.23 \\
\hline MoM-RF & 2.19 & 17.3 & 17.49 & 1.42 \\
\hline Sonnet & 2.17 & 14.09 & 16.19 & 1.57 \\
\hline CST & 2.27 & 38.7 & 16.41 & 0.6 \\
\hline \hline Meas & 2.00 & 29.8 & 18.75 & 1.11 \\
\hline
\end{tabular}

RF-CMOS process. Whereas $G_{3 D}$ predicts an inductance value that is closer to the actual measurement (Meas), the self-resonant frequency ( $\mathrm{SRF}$ ) is better determined using MoM-RF, which is partly based on a $G_{2 D}$ scheme. Differences in $Q_{\max }$ are related to the lack of knowledge about the actual conductivity value of the substrate.

\subsection{Hybrid 2D/3D Green's function}

At this point, the question that arises is which of the two pictures, $G_{2 D}$ or $G_{3 D}$, is the correct one. The answer depends on the application under study. When the device is inserted in a matched environment (i.e. there is no geometric/material change at its reference planes), and it is studied at high frequencies, a $G_{2 D}$ approach will better describe the behavior of the component. On the contrary, devices inserted in non-controlled environments and having a low $\ell / \lambda$ value should be studied using $G_{3 D}$ solvers.

It is clear that the quasi-static $G_{2 D}$ and $G_{3 D}$ functions have their pros and cons. Nevertheless, it has also been shown that both pictures can be connected artificially by tuning $G_{3 D}$ through the use of calibrated ports. Using the $\ell / \lambda$ parameter, a combination of both $G_{2 D}$ and $G_{3 D}$ Green's functions is proposed as follows

$$
G\left(\bar{r}, \bar{r}^{\prime}\right)=2 \frac{\ell}{\lambda} G_{2 D}\left(\bar{r}, \bar{r}^{\prime}\right)+\left(1-2 \frac{\ell}{\lambda}\right) G_{3 D}\left(\bar{r}, \bar{r}^{\prime}\right),
$$

where $\ell / \lambda$ is taken as the weight criterion. At $\omega=0$, the contribution in Eq. 22 is only due to $G_{3 D}$; in the highfrequency regime, it is due to $G_{2 D}$. For frequencies where $\ell \geq \lambda / 2$, the associated weight is 1 for $G_{2 D}$ and 0 for $G_{3 D}$. An additional remark must be made regarding Eq. 22): it is not necessary to derive an analytical solution of $G_{2 D}$ because it can be calculated with $G_{3 D}$ using aspect ratios bigger than $1: 10^{3}$.

Fig. 12 shows the behavior of the quality factor $\mathrm{Q}$ for the previous microstrip line using Eq. 22. The curve is bounded between the $G_{2 D}$ and $G_{3 D}$ solutions. At low and medium frequencies, the solution is closer to $G_{3 D}$; thus, the quality factor is not overestimated due to the correct computation of eddy currents in metal regions [17. Looking at the inset in Fig. 12, the behavior at resonance is closer to the $G_{2 D}$ solution. Based on this plot, Eq. 22 is a plausible GF for extending the frequency range of quasi-static solvers.

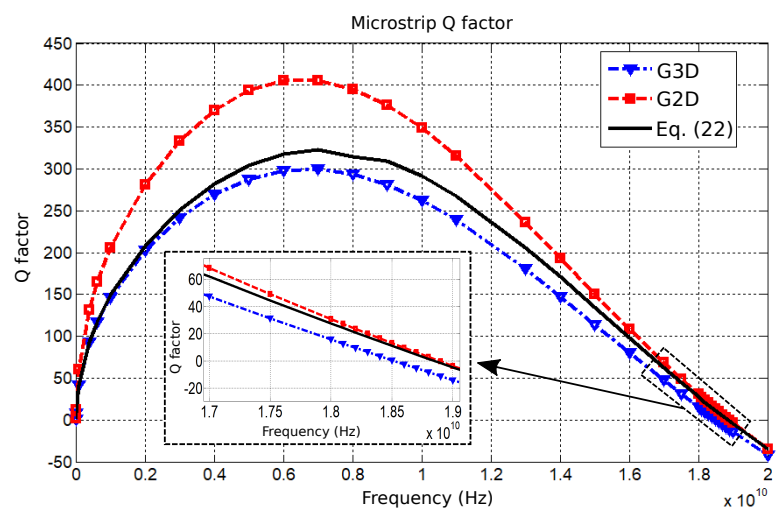

Fig. 12: Microstrip line quality factor using different GFs.

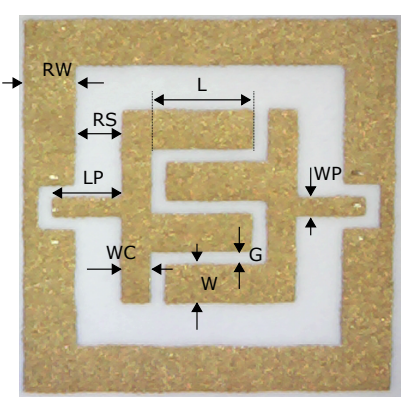

(a)

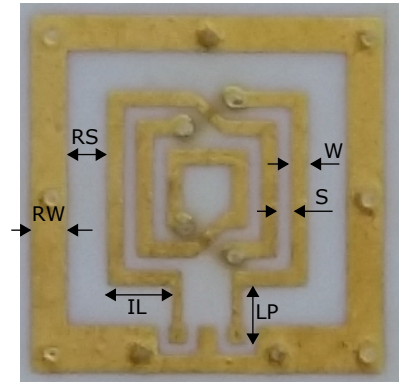

(b)
Fig. 13: Photograph of the RF devices implemented in LTCC: (a) Finger capacitor, (b) inductor.

\section{Application to $\mathrm{RF}$ and $\mathrm{uW}$ passives}

To sum up the different steps that integrate the implemented solver, it is possible to simulate the behavior of 2.5D planar devices, geometrically described by Manhattanlike layouts, placed on any metal level of a multilayered substrate with homogeneous LTI dielectric material, and under the limitation of the frequency range given by Eq. 17). The dispersion and temperature dependence of the materials can also be included in the model. The best way to demonstrate its performance is through comparison with experimental measurements (Meas) of different devices. To this end, two RF devices, a finger capacitor and an inductor, were fabricated using a low temperature co-fired ceramic (LTCC) technology, and a microwave filter was fabricated in a printed circuit board (PCB).

\subsection{RF devices in LTCC technology}

Fig. 13 shows one of the fabricated RF devices and the details of its geometric parameters are presented in Table 4. The substrate has a typical relative permittivity of 6.5 , a loss tangent lower than 0.002 and a total thickness of $840 \mu \mathrm{m}$.

For the finger capacitor, measurements were performed from $10 \mathrm{MHz}$ to $8.5 \mathrm{GHz}$, using a ground-signal-ground (GSG) $500 \mu \mathrm{m}$ probe, and a SOLT calibration procedure. 
Table 4: Geometric parameters of LTCC devices in $[\mu \mathrm{m}]$.

\begin{tabular}{l|c|c|c|c|c|c|c|c}
\hline Device & \multicolumn{10}{|c}{ Parameter } \\
\hline $\begin{array}{l}\text { Finger } \\
\text { capacitor }\end{array}$ & $\mathrm{L}$ & $\mathrm{W}$ & $\mathrm{G}$ & $\mathrm{LP}$ & $\mathrm{WP}$ & $\mathrm{WC}$ & $\mathrm{RS}$ & $\mathrm{RW}$ \\
\cline { 2 - 9 } & 1400 & 600 & 200 & 950 & 300 & 400 & 650 & 700 \\
\hline \hline \multirow{2}{*}{ Inductor } & $\mathrm{IL}$ & $\mathrm{W}$ & $\mathrm{S}$ & $\mathrm{RW}$ & $\mathrm{RS}$ & & & \\
\cline { 2 - 9 } & 1035 & 270 & 230 & 625 & 700 & & & \\
\hline
\end{tabular}

Table 5: Measured vs. simulated data of finger capacitor.

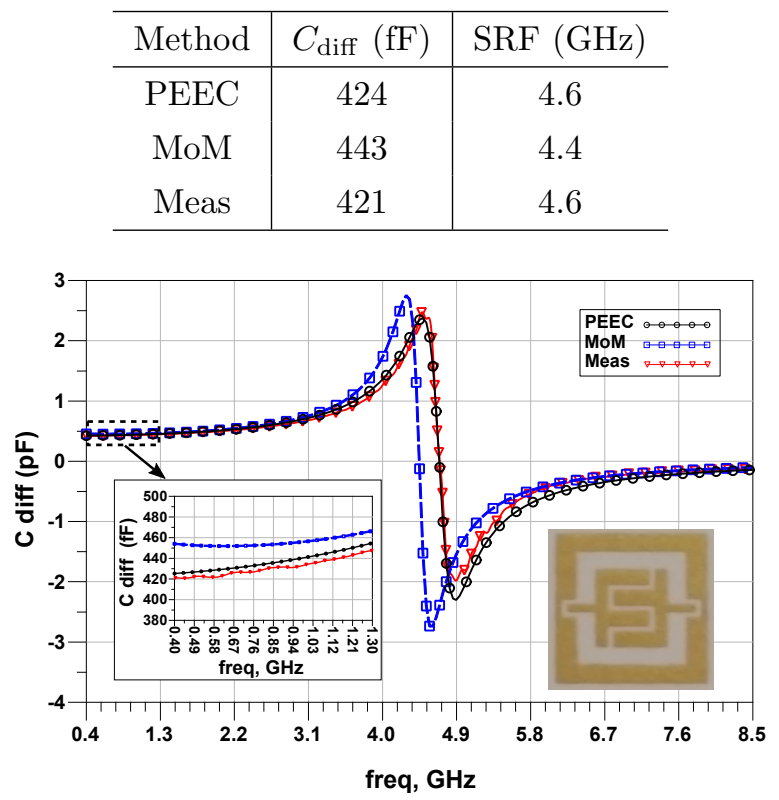

Fig. 14: Measured vs. simulated data of a finger capacitor.

The obtained values of the electrical parameters are shown in Table 5 . Notice that the estimates of the differential capacitance value, $C_{\text {diff }}$, and $\mathrm{SRF}$ are quite accurate in both PEEC and MoM-RF, with PEEC having a better match. In addition, the PEEC solver is 5 times faster than MoM-RF. When the PEEC method is implemented with a 3D-GF instead of Eq. 22, the error in SRF is larger than 50\% (not shown in Table 5).

To check the performance over a wider frequency range, Fig. 14 shows a comparison of the measured $C_{\text {diff }}$ against different solvers. The implemented PEEC method using Eq. (22) has a better accuracy because, although the frequency is quite high, there is still a non-residual effect of the $G_{3 D}$ term which accounts for magnetic/electrical couplings of the different metal strips of the device. Moreover, the inset in Fig. 14 shows that the predicted low frequency $C_{\text {diff }}$ value has a better match in the PEEC solver due to the better accuracy of the $G_{3 D}$ in the low frequency range. Thanks to the predictive mesh generator, the correct computation of losses helps to achieve this matched behavior.

For the inductor, measurements were performed from $50 \mathrm{MHz}$ to $5 \mathrm{GHz}$, using a GSGSG $500 \mu \mathrm{m}$ probe, and a SOLT calibration procedure. The obtained values of the electrical parameters are shown in Table 6, and the frequency behavior is shown in Fig. 15. The quality factor $Q$ of the inductor is well reproduced with the imple-
Table 6: Measured vs. simulated data of inductor.

\begin{tabular}{c|c|c|c}
\hline Method & $L_{D C}(\mathrm{nH})$ & $Q_{\max }$ & SRF $(\mathrm{GHz})$ \\
\hline PEEC & 17.41 & 42.32 & 2.23 \\
MoM & 16.37 & 69.81 & 2.27 \\
Meas & 17.67 & 42.66 & 2.23 \\
\hline
\end{tabular}

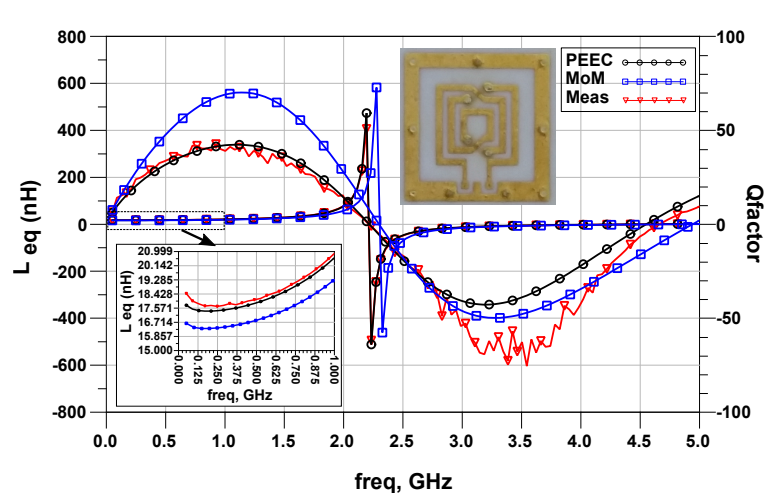

Fig. 15: Measured vs. simulated data of an inductor with guard-ring connected to GND using vias.

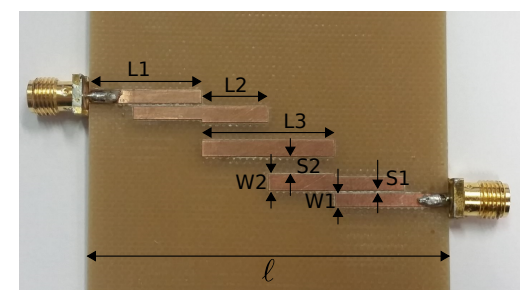

Fig. 16: Photograph of the filter implemented in PCB.

Table 7: Geometric parameters of PCB filter.

\begin{tabular}{c|c|c|c|c}
\hline Parameter & L1 & L2 & L3 & W1 \\
\hline Value $(\mathrm{mm})$ & 19.547 & 11.55 & 23.1 & 2.223 \\
\hline \hline Parameter & $\mathrm{W} 2$ & $\mathrm{~S} 1$ & $\mathrm{~S} 2$ & $\ell$ \\
\hline Value $(\mathrm{mm})$ & 2.749 & 0.433 & 2.815 & 62.194 \\
\hline
\end{tabular}

mented solver thanks to its physics-aware mesh generator, as already explained in Section 3.1. The low frequency behavior for the equivalent inductance value, represented in the inset of Fig. 15, shows a good matching between the PEEC solver and the measurements. The computation time is more than 100 times faster in PEEC than MoM-RF due to the use of high aspect ratio elements.

\subsection{Microwave filter in $P C B$ technology}

Fig. 16 shows the fabricated microwave filter and the details of its geometric parameters are presented in Table 7. The substrate has a typical relative permittivity of 4.7 , a loss tangent of about 0.013 and a substrate thickness equal to $1500 \mu \mathrm{m}$.

Measurements were performed from $2 \mathrm{GHz}$ to $5 \mathrm{GHz}$ using SMA coaxial connectors. The calibration procedure was automated using an electronic calibration kit and the 


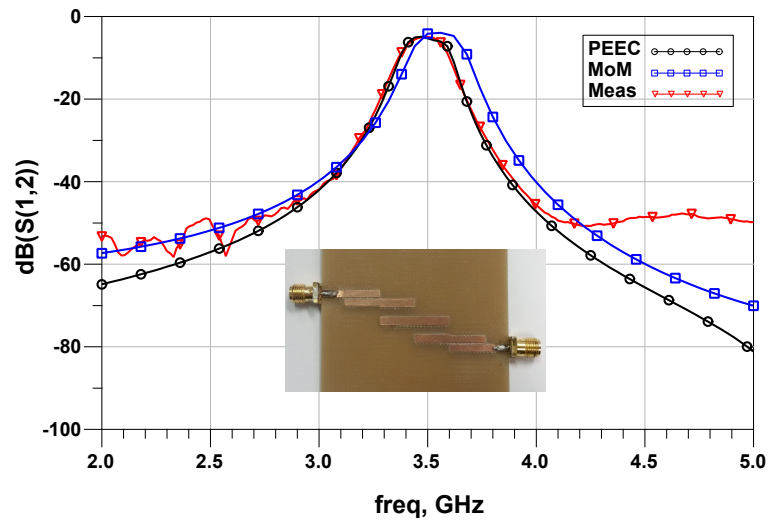

Fig. 17: Measured vs. simulated data of microwave filter.

connector influence was de-embedded by using the measurements of a short, an open and a $50 \Omega$ load inserted in a PCB. At the $3.5 \mathrm{GHz}$ pass-band frequency of the filter, the ratio $\ell / \lambda$ is 1.5 , which is much higher than the quasistatic approximation, i.e., $\ell / \lambda<0.05$. Despite this ratio, the predicted behavior with the PEEC solver shows a good accuracy in the determination of the pass-band, as shown in Fig. 17, thanks to the hybrid 2D/3D Green's function of Eq. (22). It is worth noting that the insertion loss matches well because the current mesh was generated according to the predicted influence of the skin and proximity effects.

\section{Conclusion}

This work has demonstrated a practical implementation of a quasi-static PEEC solver that relies on the generation of an optimal mesh and the evaluation of Green's function using analytical expressions. The ab initio meshing techniques developed herein demonstrated good description of losses. Moreover, the linear weighted combination of 2D and 3D quasi-static Green's function enabled the extension of the frequency range up to the onset of the first high-order wave-guide mode; thus, microwave devices can be simulated beyond the quasi-static regime $\ell / \lambda<0.05$.

\section{References}

[1] A. M. Niknejad, R. G. Meyer, Analysis of eddy-current losses over conductive substrates with applications to monolithic inductors and transformers, IEEE Transactions on Microwave Theory and Techniques 49 (1) (2001) 166-176.

[2] G. Manetas, V. N. Kourkoulos, A. C. Cangellaris, Investigation on the Frequency Range of Validity of Electroquasistatic RC Models for Semiconductor Substrate Coupling Modeling, IEEE Transactions on Electromagnetic Compatibility 49 (3) (2007) 577-584.

[3] A. E. Ruehli, Equivalent Circuit Models for Three-Dimensional Multiconductor Systems, IEEE Transactions on Microwave Theory and Techniques 22 (3) (1974) 216-221.

[4] P. Scholz, Analysis and Numerical Modeling of Inductively Coupled Antenna Systems, Ph.D. thesis, Technische Universität, Darmstadt (December 2010).

[5] D. Daroui, J. Ekman, Performance analysis of parallel nonorthogonal PEEC-based solver for EMC applications, Progress In Electromagnetics Research B (41) (2012) 77-100.
[6] G. Antonini, A. Orlandi, A. Ruehli, J. Ekman, J.Delsing, Peec development road map 2008

URL http://http://staff.www.1tu.se/ jekman/PEEC/PEEC_ Roadmap_2008.pdf

[7] S. Ahyoune, J. Sieiro, T. Carrasco, N. Vidal, J. M. LpezVillegas, E. Roca, F. V. Fernndez, Extending the frequency range of quasi-static electromagnetic solvers, in: 2017 14th International Conference on Synthesis, Modeling, Analysis and Simulation Methods and Applications to Circuit Design (SMACD), 2017, pp. 1-4.

[8] A. Weisshaar, H. Lan, A. Luoh, Accurate closed-form expressions for the frequency-dependent line parameters of on-chip interconnects on lossy silicon substrate, IEEE Transactions on Advanced Packaging 25 (2) (2002) 288-296.

[9] C. Xu, N. Srivastava, R. Suaya, K. Banerjee, Fast HighFrequency Impedance Extraction of Horizontal Interconnects and Inductors in 3-D ICs With Multiple Substrates, IEEE Transactions on Computer-Aided Design of Integrated Circuits and Systems 31 (11) (2012) 1698-1710.

[10] S. Ahyoune, Heterogeneous Integration of RF and Microwave Systems. Using Multi-layer Low-Temperature Co-fired Ceramics Technology, Ph.D. thesis, Universitat de Barcelona (Jul. 2017).

[11] K. Umetani, J. Imaoka, M. Yamamoto, S. Arimura, T. Hirano, Evaluation of the lagrangian method for deriving equivalent circuits of integrated magnetic components: A case study using the integrated winding coupled inductor, IEEE Transactions on Industry Applications 51 (1) (2015) 547-555.

[12] D. Mayer, Hamiltons principle and electric circuits theory, Advances in Electrical and Electronic Engineering 5 (1) (2006) $185-189$.

[13] S. Ahyoune, J. Sieiro, M. N. Vidal, J. M. Lpez-Villegas, F. Ramos, 1d/2d hybrid mesh peec solver for the analysis of multilayer planar circuits, in: 2017 IEEE MTT-S International Conference on Numerical Electromagnetic and Multiphysics Modeling and Optimization for RF, Microwave, and Terahertz Applications (NEMO), 2017, pp. 49-51.

[14] G. Antonini, A. Orlandi, C. R. Paul, Internal impedance of conductors of rectangular cross section, IEEE Transactions on Microwave Theory and Techniques 47 (7) (1999) 979-985.

[15] A. E. Ruehli, G. Antonini, L. J. Jiang, Skin-effect loss models for time- and frequency-domain peec solver, Proceedings of the IEEE 101 (2) (2013) 451-472.

[16] J. C. Rautio, V. Demir, Microstrip conductor loss models for electromagnetic analysis, IEEE Transactions on Microwave Theory and Techniques 51 (3) (2003) 915-921.

[17] J. Sieiro, J. M. Lpez-Villegas, M. N. Vidal, J. A. Osorio, T. Carrasco, S. Ahyoune, Ab initio adaptive meshing for planar passive component modeling, in: 2012 42nd European Microwave Conference, 2012, pp. 791-794.

[18] J. Ekman, G. Antonini, A. Orlandi, A. E. Ruehli, Impact of partial element accuracy on PEEC model stability, IEEE Transactions on Electromagnetic Compability 48 (1) (2006) 127-137.

[19] C. Hoer, C. Love, Exact inductance equations for rectangular conductors with applications to more complicated geometries, J. Res. Natl. Bur. Stand. C 62 (2) (1965) 127-137.

[20] G. Vendelin, Limitations on stripline q, Microwave Journal 13 (1970) 63-69.

[21] Momentum 3D Planar EM Simulator. [Online], accessed:1March- 2017. URL http://www . keysight .com

[22] Sonnet User's Guide. [Online] accessed:1-March- 2017. URL http://www . sonnet software.com

[23] CST Microwave Studio. [Online], accessed:1-March- 2017. URL https://www.cst.com/ 